\title{
Hearing Protection System by Using a Simple Noise Reduction Strategy
}

\author{
Izziddien Alsogkier \\ Department of Electrical and Computer Engineering, College of Engineering, Elmergib \\ University, Libya
}

DOI: https://doi.org/10.21467/proceedings.2.12

* Corresponding author email: izziddien@yahoo.de

\begin{abstract}
In this paper, a very brief discussion and analysis of the noise problem are given particularly at the work environment, where the workers hearing system needs to be protected from an excessively loud noise source but at the same time the workers need to communicate with each other. Therefore, a simple strategy of noise reduction is introduced by means of isolation plus a noise filtered communication channel to keep the worker in contact with the work environment as well as the other co-workers with a minimum noise intervention as possible.

Keywords: Noise Reduction, Noise Isolation, Noise Filtering, Passive and Active Noise Control.
\end{abstract}

\section{Introduction}

There are a lot of noise sources that disturb the workers in the work environment, for example, machines driven by internal combustion engines such as cars, trucks and tractors also tool machines like generators, air compressors, drilling, cutting machines ... etc. Moreover, heavy machineries in industrial fabrics generate very loud noise so that the workers or the operators need to be isolated but at the same time they need to communicate with each other. The same instance can be found in civil and military aviation like jet engine aircrafts particularly with propellers as helicopters, where the pilot, copilot and the passengers need to be protected and isolated from the excessive noise so that they can easily communicate with each other.

Therefore, the strategy is mainly to protect the workers or the operators' hearing system from the loud noise generated in the workplace, as well as, providing a communication channel with a minimum possible noise intervention. This, in most circumstances, is a very critical and fatal safety issue. Usually, the noise problem take place or can be defined, whenever there are a noise source, which generates the noise, noise transmission or propagation medium, where the noise travels and expands, and noise sink, which is the target element that gets affected or disturbed by the noise. Therefore, the noise problem can primarily be solved from the very beginning at the noise source which gives a very general and global solution to the problem, but this can be in some circumstances the most expensive solution (e.g. internal combustion engine exhaust system). Furthermore, a secondary solution can be done by preventing the noise to reach the

(C) 2018 Copyright held by the author(s). Published by AIJR Publisher in Proceedings of First Conference for Engineering

Sciences and Technology (CEST-2018), September 25-27, 2018, vol. 1.
This is an open access article under Creative Commons Attribution-NonCommercial 4.0 International (CC BY-NC 4.0)

A iR license, which permits any non-commercial use, distribution, adaptation, and reproduction in any medium, as long as the original work is properly cited. ISBN: $978-81-936820-5-0$ 
Hearing Protection System by Using a Simple Noise Reduction Strategy

noise sink by isolating the noise source from the noise sink this gives more local solution to the problem, but this can be in some circumstances the less expensive one.

Generally in the literature, there are two distinct classifications of noise control algorithms, which are passive and active noise control algorithms, passive is by implementing passive elements like isolating or absorbing materials to reduce the impact of the noise on the medium [1], active is by using active elements to generate an anti-noise to counteract the noise and reduce its impact on the targeted medium [2]. Moreover, sometimes in order to get maximum results, hybrid algorithms, a combination of passive and active methods can also be applied simultaneously [2].

In the following, a brief introduction to passive and active noise control techniques is given in Section 2, then the problem formulation of this paper and the experimental setup are given in Section 3. Experimental examples are presented in Subsection 3.2. Moreover, results analysis of the experiments and comments are given in section 4. Finally, conclusions and further work suggestions are given in Section 5.

\section{Passive and Active Noise Control Techniques}

Passive noise control technique is usually applied by using passive isolating and filtering elements. For example, noise isolation can be done by using sound isolating and absorbing (soundproof) materials like in civil buildings or an isolation compartment (as the case of cars, helicopter, bulldozer like machine) or at very smaller scale by implementing an isolating ear plugs, muffler headset or helmet, see Figure 1.

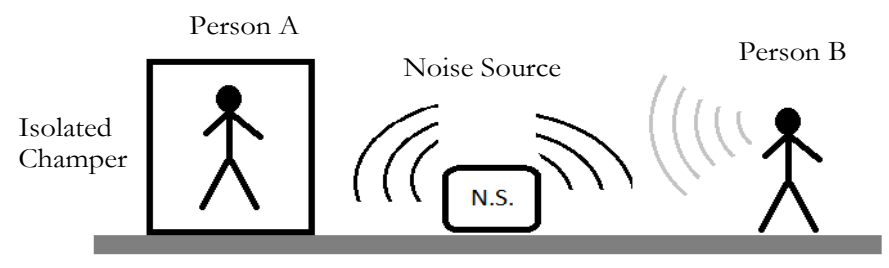

Figure 1: Passive noise control by using isolation.

The positive thing about this way of fighting the noise is that, it is very simple technically. On the other hand, apart from using intensive materials that leads to heavy weight solutions, which makes a big crucial problem particularly in the case of light weight applications like airplanes where the weight is an important factor. The negative thing about this technique is that the perfect isolation of an operator at a working place means that he will no longer receives information form the surrounding working place, which could be very fatal in most circumstances.

For example, in the case of helicopter where the pilot and the copilot need to be isolated from the excessive noise generated from the engine and the rotating blades but at the same time they need to communicate with each other without noise. This problem can be solved by 
using the setup shown in Figure 2 where both persons A and B are completely isolated from the noise and talk to each other via an isolated communication channel. This can be implemented also by using a soundproof helmet for each person with an electronic communication channel, as shown in Figure 3. Much simpler is by using ear protection system (ear muffs plus a communication system).

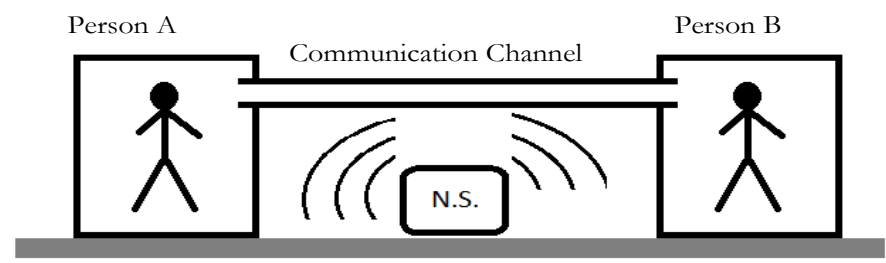

Figure 2: Two persons passive noise isolation with communication channel.

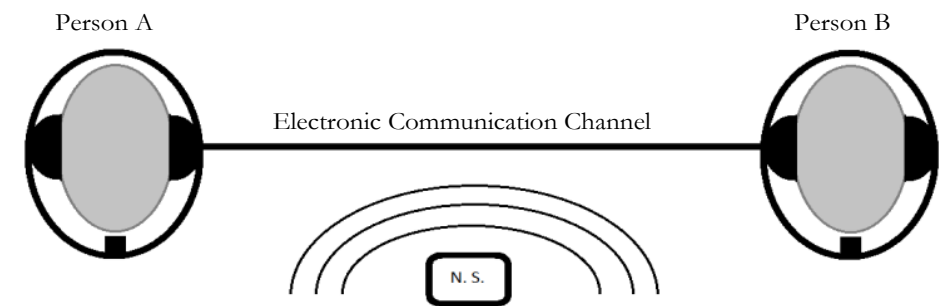

Figure 3: Two persons with head passive noise isolation and electronic communication channel.

Another example is the case of operators work beside a very loud noisy machine like an air compressor, internal combustion engine generator or any type of noisy rotational tool machines. Where, the communication channel could also be wireless based system so that the persons can move freely in their working area without any constant wire connection between them.

More specific situation regarded in this paper is when the workers need to be isolated but at the same time they need to communicate with each other. This can be done as in the helicopter case but with less expensive and simple technology by placing the operator in an isolated compartment, as shown in Figure 4, but still he receives noise filtered information from outside the isolated compartment, the communication channel is not noise isolated. Further simplification can also be achieved by using helmet or a headset ear protection system with noise filter set up as shown in Figure 5.

Alternatively, Active Noise and Control (ANC) is a technique that fights back the noise source by generating a counter action (anti-noise) against the noise actively [2]. In other words, it generates a counter action against the noise that cancels completely or partially the impact of the noise on the targeted environment. For example, in case of Figure 1, first the passive 
Hearing Protection System by Using a Simple Noise Reduction Strategy

vibration control methods can be used to reduce the vibration generated at the source, where these vibrations are the source of the noise. Moreover, the ANC methods can be applied to cancel the noise generated from the noise source. Furthermore, ANC methods can be used to cancel actively the rest noise that penetrates the non-perfect isolation compartment as demonstrated in Figure 6 and the headset stereo active noise control as shown in Figure 7. There are a lot of robust and adaptive algorithms developed for active noise and vibration control applications, see for example [3-6].

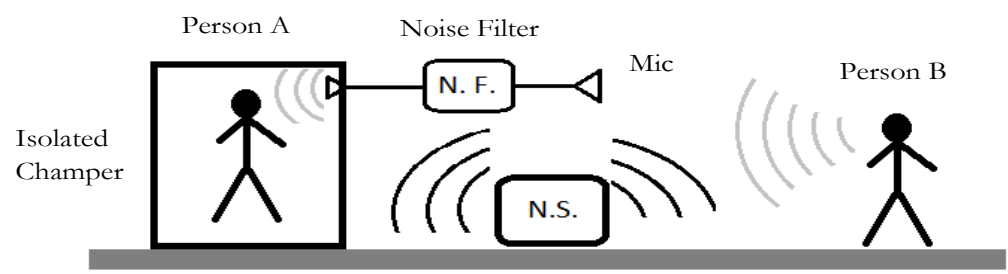

Figure 4: Passive and active noise isolation.

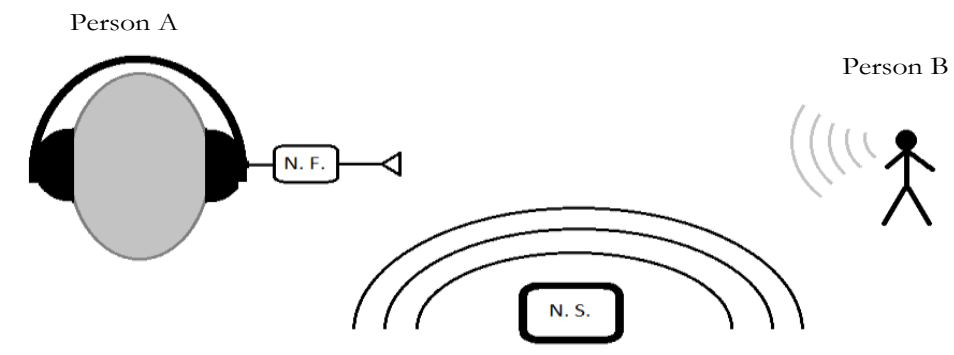

Figure 5: Passive and active noise control by using headset.

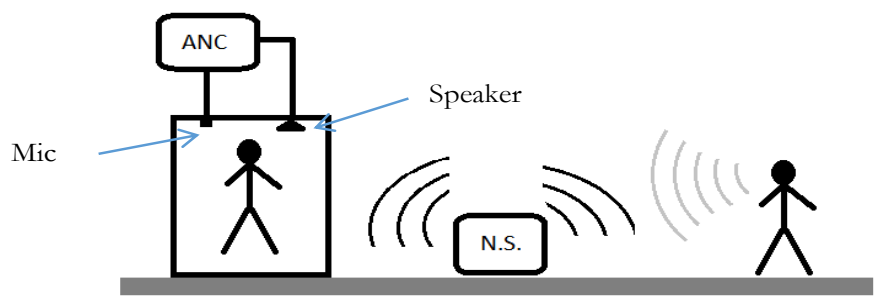

Figure 6: Active noise control system.

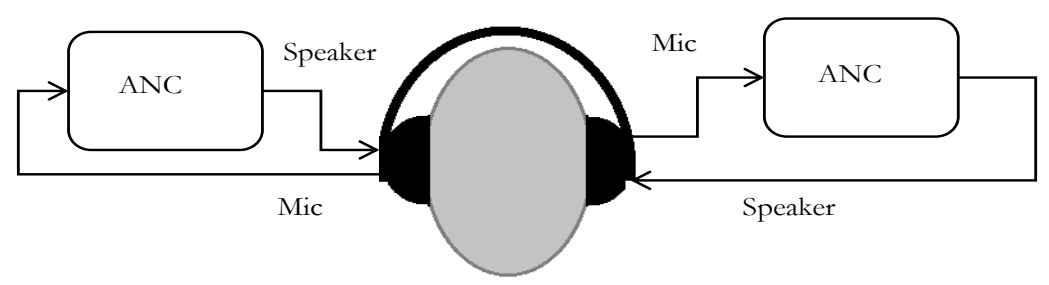

Figure 7: Stereo active noise control headset system. 


\section{Problem Formulation and Experimental Setup}

As explained in sections ( 1 and 2), it is assumed that there is a noise source that disturbs the operators or the workers in the workplace, the goal is to reduce the effect of the noise by using passive isolation as well as keeping the worker in contact with the other coworkers but with the possible minimum noise intervention. Therefore, the experiment setup will be as presented in Figure 4 and Figure 5. Moreover, the noise source considered in this experiment is assumed to be a single harmonic (sinusoidal) function. Therefore, a narrow band stop filter can be used as a noise filter to reduce the noise in the communication channel.

In this experiment, a notch filter will be designed and applied as narrow band stop filter. The notch filter can be implemented by using analog passive elements, a combination of $\mathrm{R}, \mathrm{L}$ and C circuits, or active filters by using some sort of linear amplifiers with RLC elements. Furthermore, a digital filter can also be designed and implemented by utilizing a digital signal processor.

\subsection{Notch Filter}

In this work, an analog notch filter in form of second order transfer function is designed and applied to filter out a single harmonic noise source. Hence, the notch filter transfer function is defined by

$\mathrm{G}_{\mathrm{N}}(\mathrm{s})=\frac{\mathrm{s}^{2}+2 \xi_{\mathrm{N}} \omega_{\mathrm{N}} \mathrm{s}+\omega_{\mathrm{N}}^{2}}{\mathrm{~s}^{2}+2 \xi_{\mathrm{D}} \omega_{\mathrm{D}} \mathrm{s}+\omega_{\mathrm{D}}^{2}}$

where $\omega_{N}=\omega_{D}=\omega_{\text {Notch }}$ is the notch angular frequency, $\xi_{N}=0$, or $\approx 0$ and $\xi_{D} \geq 1$.

Therefore, the notch filter transfer function becomes

$G_{N}(s)=\frac{s^{2}+\omega_{N}^{2}}{s^{2}+2 \xi_{D} \omega_{D} s+\omega_{D}^{2}}$

The following Figure 8 shows the frequency response of the notch filter for the parameters

$\omega_{\text {Notch }}=100[\mathrm{rad} / \mathrm{s}], \xi_{\mathrm{N}}=0,0.1,0.01$ and $\xi_{\mathrm{D}}=1$.

\subsection{Notch Filter Experiment}

The experimental setup as mentioned earlier is as shown in Figure 4 and Figure 5, where the noise source is a $100 \mathrm{~Hz}$ single harmonic (sinusoidal) function. Therefore the noise filter is an analog notch filter in form of second order transfer function, which is designed and applied to filter out noise from voice in the communication channel.

The experiment data is gathered and presented in Figure 9, where the voice signal without noise is plotted by a blue line, while the voice signal with $100 \mathrm{~Hz}$ noise is plotted by a green line and the filtered voice with noise signal is plotted by a red line.

Proceedings of First Conference for Engineering Sciences and Technology (CEST-2018), vol. 1 


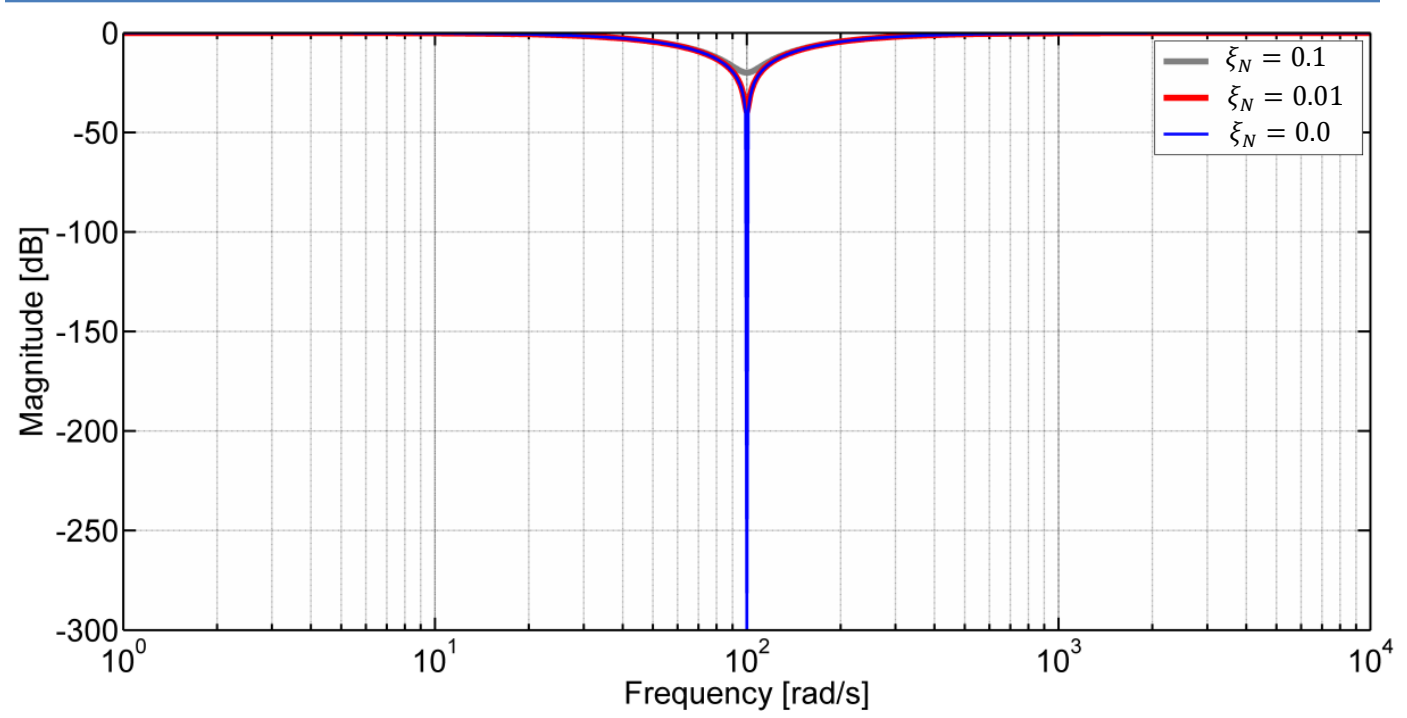

Figure 8: Notch filter freauency response.

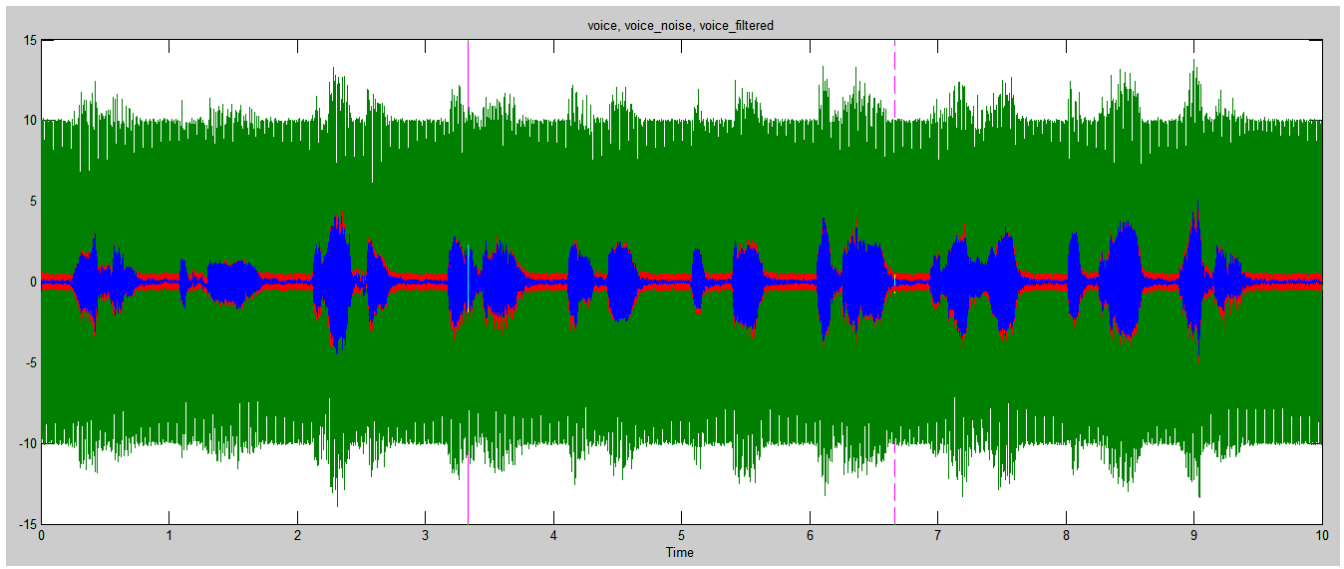

Figure 9: Original voice without noise (Blue), voice with noise (green) and filtered voice with noise (red).

\section{Results Analysis and Discussion}

Figure 9 shows the time plot of the original voice signal without noise, represented by the blue line, where the voice signal is simply a record of counting from one to ten in ten seconds. The green line is the plot of the voice signal corrupted with single harmonic noise at $100 \mathrm{~Hz}$ frequency. Moreover, Figure 10 is time axis zoom in of Figure 9 between less than 3.9 and more than 4.4 seconds, where it shows clearly the filtered voice signal still has a rest noise component but it is much better than the voice signal with noise, in fact, the filtered signal became almost like the noise free voice signal. Furthermore, Figure 11 shows the plot of the frequency spectrum of corresponding signals in Figure 9 and Figure 10 with the same 
respective colors as well. Also this graph shows that the notch filter has managed to reduce the noise component at the frequency $100 \mathrm{~Hz}$.

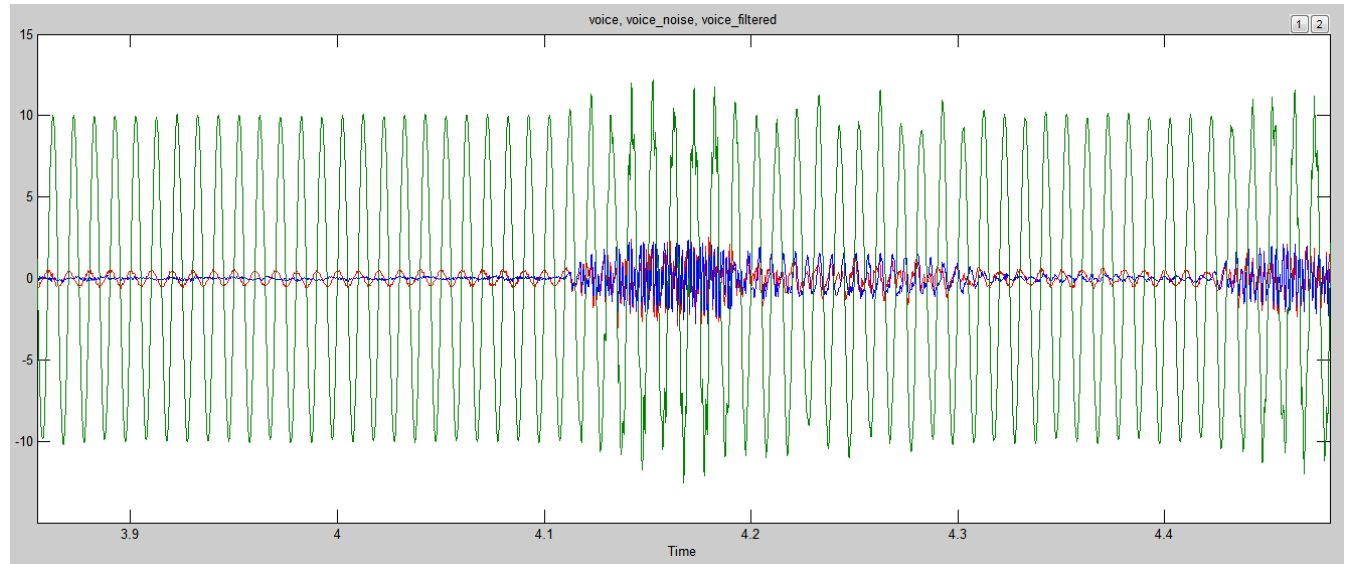

Figure 10: Original voice without noise (Blue), voice with noise (green) and filtered voice with noise (red).

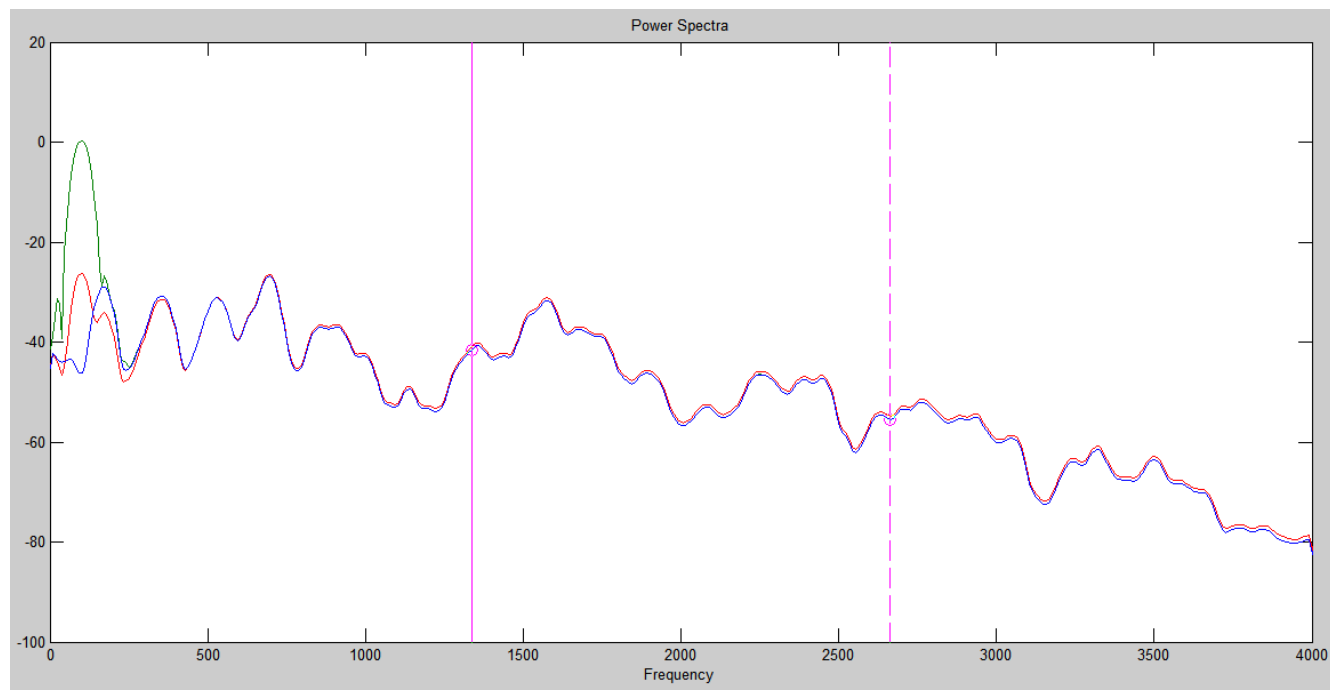

Figure 11: Frequency spectrum of original voice without noise (Blue), voice with noise (green) and filtered voice with noise (red).

\section{Conclusions and Further Works}

In this paper, instead of using isolation only to protect the workers or the operators against a very loud noise, which make them completely deaf in their working environment, a communication channel between the environment and the worker is applied with a band stop filter in form of a notch filter to reject or filter out the single harmonic noise, so that the worker can keep in contact with the work environment, this can improve the communication

Proceedings of First Conference for Engineering Sciences and Technology (CEST-2018), vol. 1 
Hearing Protection System by Using a Simple Noise Reduction Strategy

with the other coworkers as well as the environment which, in consequence, is a very important safety factor.

The application of this technique can be at its simplest, by adding (to a head set muffler) a communication channel microphone, low power audio amplifier with noise filter and very small speaker embedded in the head set muffler.

The noise filter can be designed as RLC band stop notch filter, or just an analog transfer function filter that can be built by using operational amplifiers or can be built by using a digital signal processor utilizing a digital computer system. For small scale implementations, a suitable microcontroller-based system can also be used to realize this strategy.

\section{References}

[1] I. L. Ver and L. L. Beranek, "Noise and vibration control engineering principles and applications," John Wiley \& Sons, 2006.

[2] C. H. Hansen, "Understanding active noise cancellation," Taylor \& Francis e-Library, 2003.

[3] B. Widrow and S. D. Stearns, "Adaptive signal processing," Prentice-Hall PTR, 1985.

[4] C. R. Fuller, S. J. Elliott and P. A. Nelson, "Active control of vibrations," Academic Press, 1996.

[5] S. Elliott, "Signal processing for Active Control," Academic Press, 2001.

[6] B. Widrow and E. Walach, "Adaptive inverse control a signal processing approach," John Wiley \& Sons, 2008. 